\title{
Ambipolar charge carrier transport in mixed organic layers of phthalocyanine and fullerene
}

Cite as: J. Appl. Phys. 101, 063709 (2007); https://doi.org/10.1063/1.2436836

Submitted: 11 October 2006 . Accepted: 18 December 2006 . Published Online: 27 March 2007

Andreas Opitz, Markus Bronner, and Wolfgang Brütting

\section{ARTICLES YOU MAY BE INTERESTED IN}

Electronic properties of organic semiconductor blends: Ambipolar mixtures of phthalocyanine and fullerene

Applied Physics Letters 90, 212112 (2007); https://doi.org/10.1063/1.2742640

Two-layer organic photovoltaic cell

Applied Physics Letters 48, 183 (1986); https://doi.org/10.1063/1.96937

Small molecular weight organic thin-film photodetectors and solar cells

Journal of Applied Physics 93, 3693 (2003); https://doi.org/10.1063/1.1534621

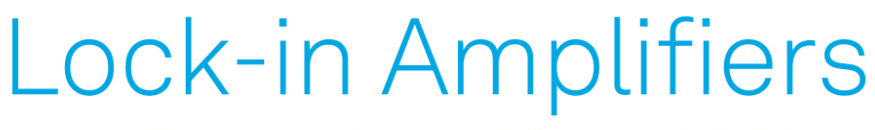

... and more, from DC to $600 \mathrm{MHz}$

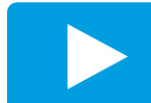

Watch 


\title{
Ambipolar charge carrier transport in mixed organic layers of phthalocyanine and fullerene
}

\author{
Andreas Opitz, ${ }^{\text {a) }}$ Markus Bronner, and Wolfgang Brütting \\ Experimentalphysik IV, Universität Augsburg, 86135 Augsburg, Germany
}

(Received 11 October 2006; accepted 18 December 2006; published online 27 March 2007)

\begin{abstract}
Mixed layers of copper-phthalocyanine ( $p$-conductive) and fullerene ( $n$-conductive) are used for the fabrication of organic field-effect transistors (OFET) and inverters. Regarding the electrical characteristics of these donor-acceptor blends they show ambipolar charge carrier transport, whereas devices made from only one of the materials show unipolar behavior. Such mixed films are model systems for ambipolar transport with adjustable field-effect mobilities for electrons and holes. By variation of the mixing ratio it is possible to balance the transport of both charge-carrier types. In this paper we discuss the variation of mobility and threshold voltage with the mixing ratio and demonstrate ambipolar inverters as a leadoff application. The gained results were analyzed by simulations using an analytical model for ambipolar transistors and subsequently compared to complementary inverters. (C) 2007 American Institute of Physics. [DOI: 10.1063/1.2436836]
\end{abstract}

\section{INTRODUCTION}

In recent years organic semiconductors have attracted considerable interest due to their growing potential as active materials in electronic and optoelectronic devices. A longstanding paradigm, however, has been seen in their unipolar transport of electrical charges. This means that, apart from very few exceptions, organic semiconductors have shown electrical conduction for one carrier species only, with positive carriers being preferentially transported in most materials. Thus multilayer structures comprising different organic materials with spatially separated electron and hole transport layers, where used for fabricating efficient organic lightemitting diodes. ${ }^{1}$ On the other hand, in photovoltaic devices, owing to short exciton diffusion length in organic semiconductors, $p$ - and $n$-conducting materials need to be in close contact which is usually realized by mixing them in one single layer yielding a so-called bulk-heterojunction structure. $^{2-6}$ Recently, such donor-acceptor mixtures have been implemented also in organic field-effect transistors (OFET) and for the first time OFET with ambipolar characteristics were demonstrated. ${ }^{7}$ Meanwhile ambipolar OFET have been realized with a variety of material combinations, including polymer/fullerene blends, ${ }^{7}$ mixtures of soluble oligomers $^{8}$ as well as evaporated molecular hetero-layer structures and mixed layers. ${ }^{9-11}$ However, even in neat films of a single organic semiconductor ambipolar transport can be observed provided that the barrier for electron injection is reduced by choosing low-work function metals as sourcedrain electrodes ${ }^{12}$ and that electron trapping at the interface to the gate-dielectric is suppressed by surface functionalization or suitable polymeric dielectrics. ${ }^{13}$ Very recently, lowband gap materials have been suggested as especially promising candidates for ambipolar OFETs. ${ }^{14}$

The successful fabrication of organic integrated circuits, which are a key feature for realizing electronics based on

\footnotetext{
a) Author to whom correspondence should be addressed; electronic mail: andreas.opitz@physik.uni-augsburg.de
}

organic semiconductors, largely relies on the properties and functions of OFET as their basic constituents. So far, only unipolar logic architectures consisting of $p$-conducting organic semiconductors have evolved into a mature technology, mainly due to the simplicity of circuit fabrication requiring only one semiconducting layer and due fairly high environmental stability of organic materials. ${ }^{15,16}$ However, unipolar logic suffers from a number of drawbacks, such as low performance, limited robustness, and high power consumption. As in silicon microelectronics, these problems in principle can be overcome by using complementary logic circuits comprising of $p$ - and $n$-channel transistors. Although, the proof-of-principle of this approach has already been demonstrated, ${ }^{17}$ the fabrication of spatially separated organic $p$ - and $n$-channel transistors in an integrated circuit with micron-sized features still appears to be a challenge. Thus, ambipolar OFET which are fabricated almost as simple as their unipolar counterparts might be of interest for realizing complementary-like organic integrated circuits. ${ }^{14}$ Moreover, it has been revealed that ambipolar OFET can provide additional functionalities as light-emitting transistors $^{18,19}$ or phototransistors.

Therefore, one intention of this paper is to compare ambipolar inverters constructed of two ambipolar transistors with their complementary counterparts consisting of two discrete $p$ - and $n$-channel transistors of the same materials. For this purpose we prepare OFET of pure and mixed semiconducting films made from copper-phthalocyanine and buckminster-fullerene with various mixing ratios and investigate their transport properties. The results are related to the characteristics of ambipolar and complimentary inverters and compared to simulations using an analytical model. We will demonstrate that balanced mobilities of electrons and holes can be achieved by varying the mixing ratio. This turns out to be a crucial requirement for both ambipolar and complementary logic devices. 
(a)

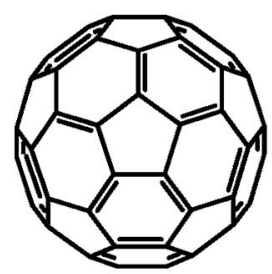

(b)
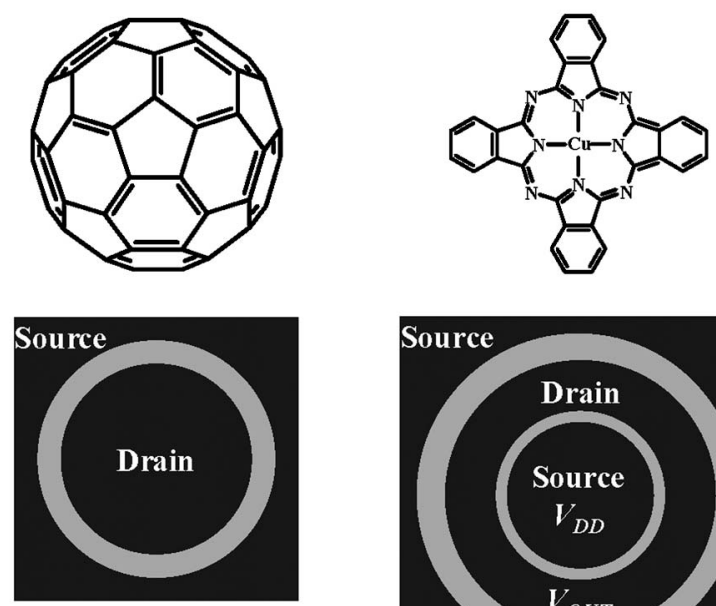

(c)

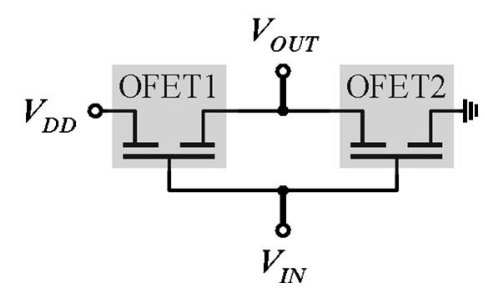

FIG. 1. (a) Chemical structures of the used materials. Left: Fullerene $\left(\mathrm{C}_{60}\right)$, right: Copper-phthalocyanine $(\mathrm{CuPc})$. (b) Top view of the ring-type transistor (left) and ring-type inverter (right). The silicon substrate acts as the gate electrode in both cases. (c) Electrical circuit of the used inverters.

\section{DEVICE PREPARATION AND EXPERIMENTAL METHODS}

Organic field-effect transistors were fabricated on highly conductive $\mathrm{Si}$ wafers $(1-5 \mathrm{~m} \Omega \mathrm{cm})$ with a 200 or $320 \mathrm{~nm}$ thick thermally grown oxide, which acts as gate insulator. Photolithographically patterned $\mathrm{Ti}(1 \mathrm{~mm}) / \mathrm{Au}(100 \mathrm{~nm})$ source and drain electrodes were made by electron-beam evaporation and by lift-off process. These structures were cleaned in an ultrasonic bath with solvents (acetone and isopropyl) and ultra-pure water. The substrates were dried with pure nitrogen, treated with an $\mathrm{O}_{2}$ plasma for $60 \mathrm{~s}$ at $200 \mathrm{~W}$ and $0.6 \mathrm{mbar}$, and heated in fore-vacuum at $400 \mathrm{~K}$ for $2 \mathrm{~h}$.

Copper-phthalocyanine ( $\mathrm{CuPc}$, purchased from Aldrich as sublimation grade $)$ and buckminster fullerene $\left(\mathrm{C}_{60}\right.$, purchased from Hoechst as super gold grade) were used as hole and electron conducting materials, respectively. The structural formulas are given in Fig. 1(a). The materials were deposited by thermal evaporation from low-temperature effusion cells in a vacuum better than $1 \times 10^{-7} \mathrm{mbar}$ to form pure and mixed layers with a thickness of about $25 \mathrm{~nm}$ on the prestructured substrates. The deposition rates were between $0.35 \AA / \mathrm{s}$ for neat films and $1.4 \AA / \mathrm{s}$ for layers with $1: 3$ stoichiometry. The given mixing ratios in this paper are volume percentages as the evaporation process was controlled via the deposition rates monitored with a quartz microbalance. They are always given in the form $\mathrm{C}_{60}$ : $\mathrm{CuPc}$. Different substrate temperatures ( 300 and $375 \mathrm{~K}$ ) were used during evaporation of the materials.

Transistors with a ring-type geometry [see Fig. 1(b), lefthand side] were used, whose source electrodes form a closed ring around the drain electrodes. This prevents parasitic currents from outside of the active transistor channel without the necessity of structuring the organic semiconductor. ${ }^{20}$ The channel length and width were 5 and $2500 \mu \mathrm{m}$, respectively. Ambipolar inverters were made from two transistors stacked into each other [see Figs. 1(b) and 1(c)] and have an additional ring channel around the first one. Thus both transistors share the common silicon substrate gate electrode as input of the inverter and the drain electrode as output. Length and width of the outer channel were 10 and $2500 \mu \mathrm{m}$, respectively, and of the inner channel 8 and $2000 \mu \mathrm{m}$, respectively. Consequently the ratio of length to width was the same for both channels. Each substrate $(20 \mathrm{~mm} \times 20 \mathrm{~mm})$ contained 24 individual transistors and 12 inverter structures to allow for a comparison of several devices. Complementary inverters were fabricated by evaporating pure $\mathrm{CuPc}$ and $\mathrm{C}_{60}$ each on one half of a prestructured substrate. For this purpose two evaporation steps were necessary with different shadow masks. In this case two separated transistors, one $p$-type and one $n$-type, from different areas on the substrate were connected together to form an inverter.

Without air-exposure the devices were transferred to a vacuum-chamber providing a pressure less than $5 \times 10^{-6}$ mbar for characterization. The output and transfer characteristics of the transistors were measured using two independent source-meter units (Keithley 236). In order to measure inverter transfer curves an additional source-meter unit (Keithley 2400) was implemented.

The charge-carrier mobilities $\mu$ and the threshold voltages $V_{T}$ were extracted from the slope of the transfer characteristics in the saturation region $\left|V_{D}\right|>\left|V_{G}-V_{T}\right|$ using the standard relationship

$$
I_{D, \text { sat }}=\frac{W}{2 L} \mu C_{O x}\left(V_{G}-V_{T}\right)^{2} .
$$

Here $W$ is the channel width, $L$ is the channel length, $C_{O x}$ is the gate-oxide capacitance per unit area, $V_{G}$ is the gate voltage, and additionally $V_{D}$ is the drain voltage. Mobility $\mu$ and threshold voltage $V_{T}$ were determined from the linear regression of the measured data plotted as $\sqrt{I_{D, \text { sat }}}$ versus $V_{G}$. The inverter characteristics include the transfer curve $V_{\text {OUT }}\left(V_{\text {IN }}\right)$ as well as the current dissipation $I_{D D}\left(V_{\mathrm{IN}}\right)$.

$\mathrm{X}$-ray diffraction (XRD) patterns were obtained in $\theta-2 \theta$ geometry using a Siemens D-500 diffractometer $(\mathrm{Cu} \mathrm{K \alpha}$ radiation with a wavelength of $0.1542 \mathrm{~nm}$ ) for analyzing the crystallinity of the films. The morphology investigations were performed using a Thermo Microscopes Auto Probe scanning force microscope (SFM) operated in noncontact (tapping) mode.

\section{RESULTS AND DISCUSSION}

\section{A. Unipolar field-effect transistors}

Output and transfer characteristics of pure $\mathrm{C}_{60}$ and $\mathrm{CuPc}$ FET are shown in Fig. 2. The output characteristics show the typical unipolar transistor behavior with linear increase at low drain voltage and saturation at higher $V_{D}$. The transfer 

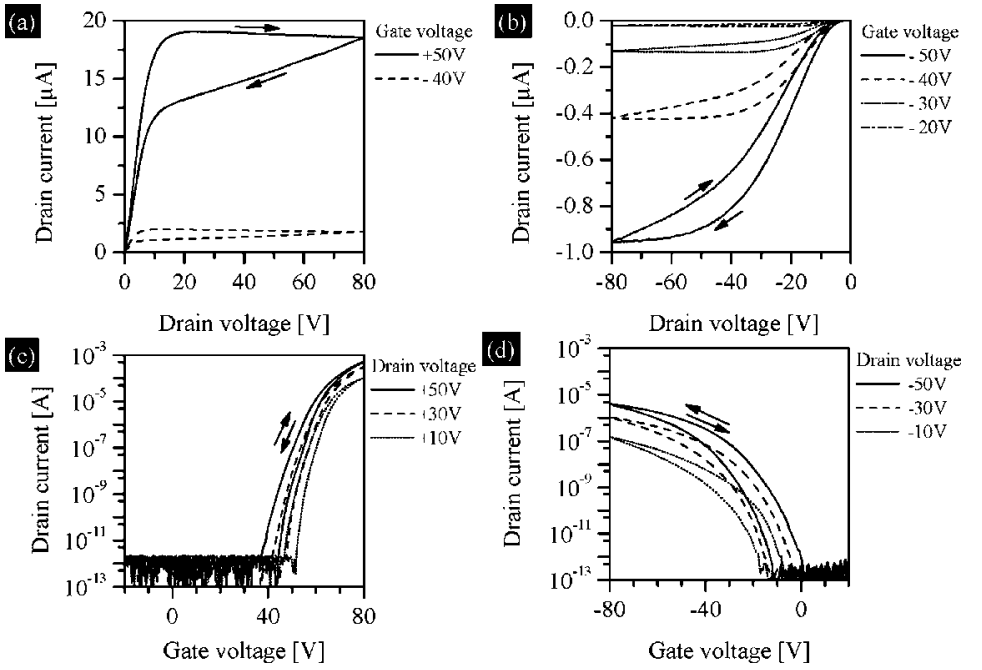

Drain voltage $[\mathrm{V}]$
FIG. 2. Output characteristics of unipolar field-effect transistors with $\mathrm{C}_{60}$ (a) and $\mathrm{CuPc}$ (b) and corresponding transfer characteristic for $\mathrm{C}_{60}(\mathrm{c})$ and for $\mathrm{CuPc}(\mathrm{d})$. The substrates were $\mathrm{O}_{2}$-plasma treated and the films evaporated at $375 \mathrm{~K}$ substrate temperature. The direction of the hysteresis is indicated by arrows. characteristics display an off-regime followed by an increase of drain current with increasing absolute value of the gate voltage exceeding the switch-on voltage.

The analysis of the transfer characteristics yielded saturation mobilities for these preparation conditions $\left(\mathrm{O}_{2}\right.$ plasma treatment, $375 \mathrm{~K}$ during evaporation) of $7 \times 10^{-2} \mathrm{~cm}^{2} / \mathrm{V} \mathrm{s}$ for the $\mathrm{C}_{60} \mathrm{FET}$ and $6 \times 10^{-4} \mathrm{~cm}^{2} / \mathrm{V}$ s for the CuPc FET. The threshold voltages were +63 and $-31 \mathrm{~V}$.

The measured $I-V$ characteristics show considerable hysteresis between increasing and decreasing voltage sweeps which can be attributed to dynamic processes in the charging of the semiconductor-dielectric interface, ${ }^{21}$ however, this will not be discussed furthermore in this paper. The largest hysteresis is present in the output characteristics of the $\mathrm{C}_{60}$ FET. The hysteresis in all other measurements, including the ambipolar FET, and in particular for the transfer characteristics which were used for the analysis, are significantly smaller.

The curvature in the output characteristics of CuPc at the origin of the $I-V$ diagram suggests an injection barrier with a nonlinear contact resistance whereas the linear increase in the $\mathrm{C}_{60}$ FET indicates a smaller injection barrier with a linear contact resistance. This is related to the electronic structure of the $\mathrm{Au} / \mathrm{CuPc}$ and the $\mathrm{Au} / \mathrm{C}_{60}$ interface. ${ }^{22-27}$ The injection barrier for electrons from gold into $\mathrm{C}_{60}$ is about $0.4 \mathrm{eV}$, whereas the value for holes into $\mathrm{CuPc}$ is significantly larger (about $0.9 \mathrm{eV}$ ).

\section{B. Ambipolar field-effect transistors}

Ambipolar FET with mixing ratios of $1: 3,1: 1$, and $3: 1$ between $\mathrm{C}_{60}$ and $\mathrm{CuPc}$ have been investigated. All have shown ambipolar transport with the same qualitative features as the 1:1 mixture displayed in Fig. 3. Here a strong increase of the current in saturation of the output characteristic [Figs. $3(\mathrm{a})$ and 3(b)] is measured for both the $n$ - and the $p$-channel regime. This is a clear signature of ambipolar behavior. Electrons are injected at higher drain voltages into the holeconducting channel, and vice versa. Consequently, the transfer characteristics [(Figs. 3(c) and 3(d)] do not show any off regime as the ambipolar increase takes place in the regime where the FET with the pure materials is switched off.

The magnitude of the drain currents in both regimes differs significantly for this mixing ratio. The $p$-channel (negative $V_{G}$ ) shows a current which is three orders of magnitude lower than the current in the $n$-channel (positive $V_{G}$ ). In both measurements the curvature in the output characteristics at the origin of the $I$ - $V$ diagram suggests injection barriers with
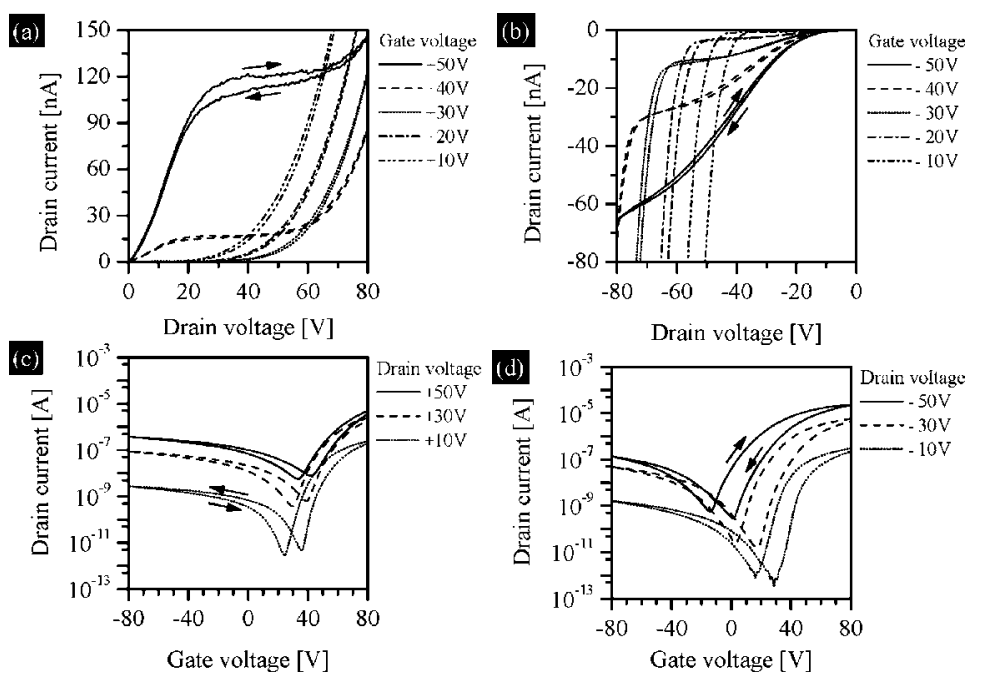

FIG. 3. Output characteristics of ambipolar field-effect transistors for a mixing ratio between $\mathrm{C}_{60}$ and $\mathrm{CuPc}$ of $1: 1$ in the $n$-channel (a) and the $p$-channel regime (b) together with the corresponding transfer characteristics in the $n$-channel (c) and the $p$-channel regime (d). The substrate was $\mathrm{O}_{2}$-plasma treated and the film was evaporated at $375 \mathrm{~K}$ substrate temperature. The direction of the hysteresis is indicated by the arrows. 

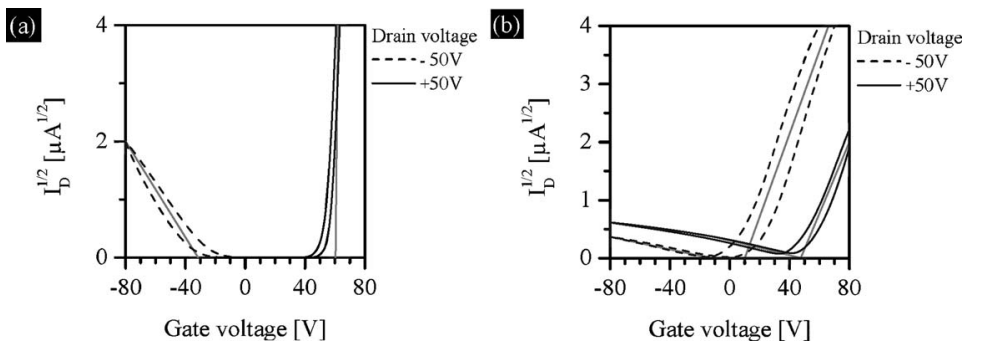

FIG. 4. The square root of the drain current vs the gate voltage of unipolar field-effect transistors with $\mathrm{C}_{60}$ and $\mathrm{CuPc}$ (a) and the film with a mixing ratio of $1: 1$ (with raw data from Figs. 2 and 3). The gray lines are linear fits from which the mobilities were determined.

nonlinear contact resistances. Here this contact resistance is also visible in the electron channel and even more pronounced in the hole channel because the conductivity of both channels is reduced due to mutual dilution of the conducting material by the other species. The nonlinear regime is then widened if the same injection barrier is assumed.

Figure 4 shows the analysis of the transfer characteristics as the square root of the drain current versus the gate voltage of films with a mixing ratio of $1: 1$ as compared to neat films of $\mathrm{C}_{60}$ and $\mathrm{CuPc}$. From the slope which is directly proportional to the square root of the mobility, a decrease of the electron and hole mobility is visible in the mixed film in comparison to the mobilities in the pure films. This is a general feature of mixed $\mathrm{CuPc} / \mathrm{C}_{60}$ layers and will be discussed in more detail in the following subsection.

\section{Dependence of charge carrier mobility on composition and thermal treatment}

Figure 5 shows the variation of electron and hole mobilities with the mixing ratio for two different substrate temperatures during evaporation (300 and $375 \mathrm{~K}$ ). Apparently, an exponential decrease of both $\mu_{e}$ and $\mu_{h}$ is observed upon dilution of the corresponding conducting material with the other species. A similar decrease has been reported in the literature for an ambipolar light-emitting system of coevaporated molecular materials. ${ }^{19}$ Furthermore, we find that heating the substrate during evaporation increases the mobilities of neat films and mixed layers by up to three orders of magnitude. Preliminary investigations have shown that a

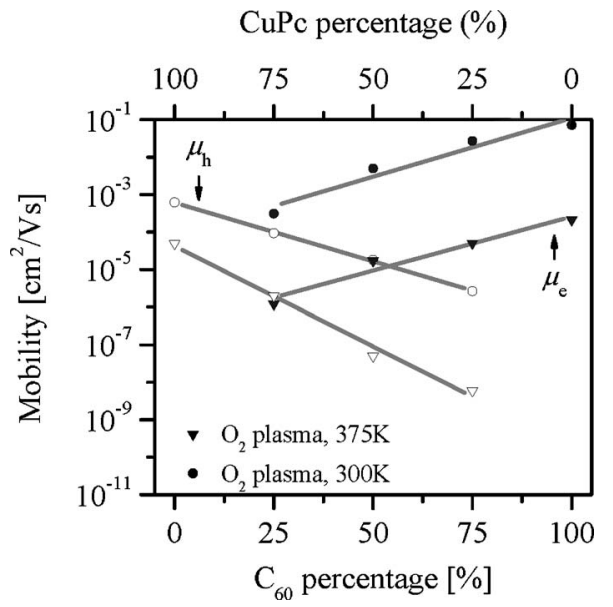

FIG. 5. Dependence of the mobility on the mixing ratio for different substrate temperatures during organic film deposition (300 and $375 \mathrm{~K}$ ). The mobilities are determined from the measurements in the saturation regime Open symbols are related to the hole mobilities and filled symbols to the electron mobilities. The gray lines are linear fits conducing to explicitness. further increase is obtained if substrate heating is combined with silanisation of the $\mathrm{SiO}_{2}$ surface prior to the deposition of the organic materials. Mobilities of up to $1 \mathrm{~cm}^{2} / \mathrm{V} \mathrm{s}$ and $2 \times 10^{-2} \mathrm{~cm}^{2} / \mathrm{V}$ s were achieved for neat $\mathrm{C}_{60}$ and $\mathrm{CuPc}$ films, respectively. Remarkably, as the data in Fig. 5 show, there is a mixing ratio where the mobilities of electrons and holes are equal. The films with balanced mobilities are CuPc-rich with about $75 \% \mathrm{CuPc}$ and $25 \% \mathrm{C}_{60}$ content, which seems to be related to the lower mobilities in neat $\mathrm{CuPc}$ films in comparison to $\mathrm{C}_{60}$ films. We note that this mixing ratio for balanced mobilities of about $3: 1$ is even valid for layers deposited on silanised $\mathrm{SiO}_{2}$, where a balanced mobility of $1 \times 10^{-2} \mathrm{~cm}^{2} / \mathrm{V} \mathrm{s}$ is achieved in this case.

In order to get more insight into the concentration dependent electron and hole mobility, structure and morphology of neat and mixed films (evaporated at $375 \mathrm{~K}$ ) were investigated by $\mathrm{x}$-ray diffraction and scanning force microscopy. XRD measurements (not displayed here) of pure CuPc films show a strong (200) peak corresponding to the $\alpha$-phase, whereas for all mixtures including pure $\mathrm{C}_{60}$ films no diffraction peaks are detectable, indicating an amorphous structure. These observations are in full agreement with measurements reported on $\mathrm{CuPc}: \mathrm{C}_{60}$ mixtures for photovoltaic applications by Rand et al. ${ }^{28}$ The authors also observed a disappearance of the CuPc diffraction peak for a fullerene content of more than about $15 \%$ and asserted no peak corresponding to $\mathrm{C}_{60}$, although, like in our case, the electron mobility in neat $\mathrm{C}_{60}$ films was about two orders of magnitude higher than the hole mobility in CuPc. Other groups have obtained crystalline $\mathrm{C}_{60}$ films, however, only at elevated substrate temperatures of about $440 \mathrm{~K}$ during evaporation. ${ }^{29}$

Figure 6 shows the surface morphologies for different mixing ratios as investigated by noncontact SFM. Neat CuPc films have a needle-like structure, corresponding to the $\alpha$-phase structure observed in XRD. For pure $\mathrm{C}_{60}$ films a granular structure is observed consisting of amorphous grains. Both films are relatively smooth with a height scale of about $8 \mathrm{~nm}$. This also holds for CuPc-rich films with a mixing ratio of $1: 3$, however, the grain size is now significantly reduced as compared to pure $\mathrm{C}_{60}$ films and smaller than the needles in pure $\mathrm{CuPc}$ films. It is worth noting that this composition has balanced mobilities for electrons and holes. The other two films with higher fullerene content (mixing ratios of $1: 1$ and $3: 1$ ) are much rougher as the height scale in the SFM images increases to 40 and $60 \mathrm{~nm}$, respectively. Consider that the nominal film thickness is only about $25 \mathrm{~nm}$. This observation can be related to demixing and phase separation between $\mathrm{C}_{60}$ and $\mathrm{CuPc}$ at elevated deposition temperature. ${ }^{30}$ 

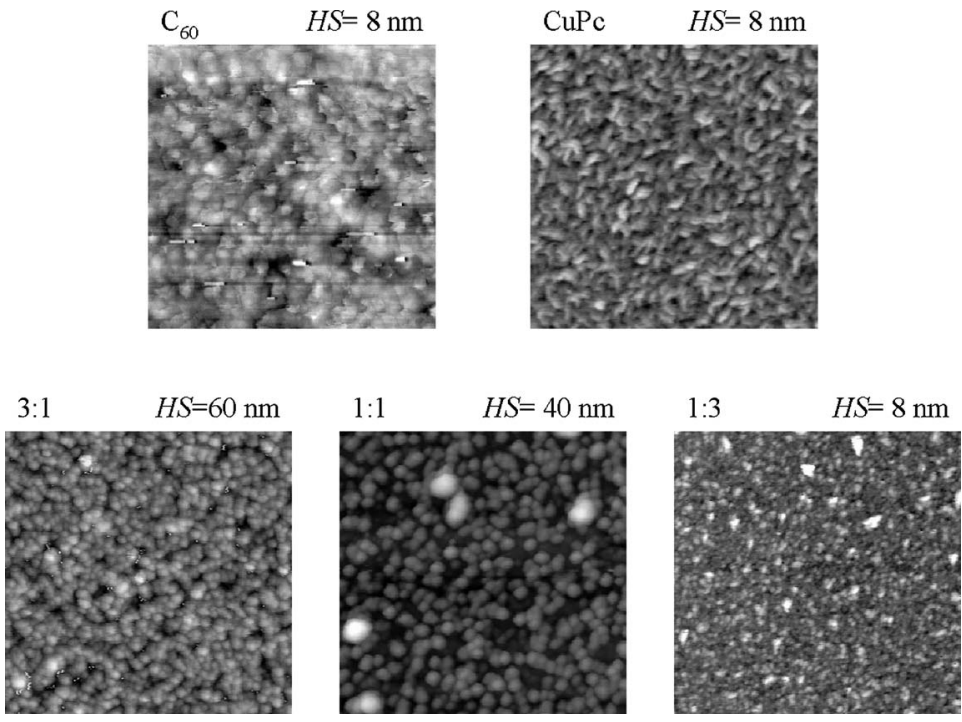

FIG. 6. Scanning force microscopy images taken in noncontact mode for pure $\mathrm{C}_{60}$ and $\mathrm{CuPc}$ films as well as for three mixed films grown at $375 \mathrm{~K}$. The total image size is $1.9 \times 1.9 \mu \mathrm{m}^{2}$. The height scale (HS) is given as the difference between the lowest value (black) and the highest value (white) in each of the images.
At this point it is noteworthy to compare our results on the variation of charge carrier mobilities with composition of the blends to other ambipolar mixed systems. There are, on the one hand, polymer-fullerene blends used for photovoltaic applications (in particular MDMO-PPV:PCBM) $)^{31,32}$ On the other hand blends of molecular materials implemented again in photovoltaic devices (mostly phthalocyanine: $\left.\mathrm{C}_{60}\right)^{28}$ and ambipolar light-emitting transistors. ${ }^{33}$ In both types of blend systems one observes, as a common feature, a strong (in many cases exponential) decrease of the mobility of one carrier type with increasing dilution by the other component. A remarkable exception from this rule is the hole mobility in polymer-fullerene blends in which the addition of fullerene molecules even improves $\mu_{h}$. In this case a stretching of the polymer chains is discoverable which can be put down to the presence of PCBM leading to improved interchain hopping. ${ }^{32}$ There is not much of an interaction among materials in low-molecular blends, they rather tend to dilute each other. The fact that the average distance between molecules of the same type in mixed donor-acceptor films is slightly increased leads to a reduction of hopping probability and thereby compared to neat films of one material only also to a reduction of the charge carrier mobility. It is remarkable, however, that despite of a roughness which is larger than the film thickness in some of our films, all of them show $n$-channel as well as $p$-channel conductivity. Obviously, there still exists a percolation path for conduction in both materials independent of phase separation and order formation. We also note that the bulk morphology does not necessarily provide precise information for organic field-effect transistors since in these devices the active channel is restricted to the first few molecular layers at the interface to the gate dielectric. ${ }^{34}$ Nevertheless our results are conformed to the bulk mobility measurements by Rand $e t$ al. ${ }^{28}$

Figure 7 shows the threshold voltages in dependence on the mixing ratio and the substrate temperature (300 and 375 K) during evaporation of the organic films. For both regimes ( $n$ - and $p$-channel), the threshold voltage has the most positive value at a mixing ratio of $1: 1$. Also, there is no significant difference upon thermal treatment. Apparently, the change of the threshold voltages is stronger for the $p$-channel than for the $n$-channel regime. A possible explanation can be found in the nature of $\mathrm{CuPc} / \mathrm{C}_{60}$ blends being donor-acceptor systems. Measurements by photoelectron spectroscopy ${ }^{25}$ have shown that there is an electron transfer from $\mathrm{CuPc}$ to $\mathrm{C}_{60}$ leading to band bending with hole accumulation at the $\mathrm{CuPc}$ side of the $\mathrm{CuPc} / \mathrm{C}_{60}$ interface, whereas no band bending arises at the $\mathrm{C}_{60}$ side. Although the reason for this has not yet been worked out in detail, the hole accumulation in $\mathrm{CuPc}$ at the organic-organic interface would explain a more positive threshold voltage for the $n$-channel regime as can be seen in Fig. 7. ${ }^{9}$ Furthermore, it is plausible that the highest charge transfer occurs at the mixing ratio of $1: 1$ where the largest organic-organic interface area exists. The threshold voltage shift will decrease in accordance with the interfacial area for increasing fraction of one of the two materials. Because the hole accumulation is on the $\mathrm{CuPc}$ side of the organic-organic interface and there is no electron accumula-

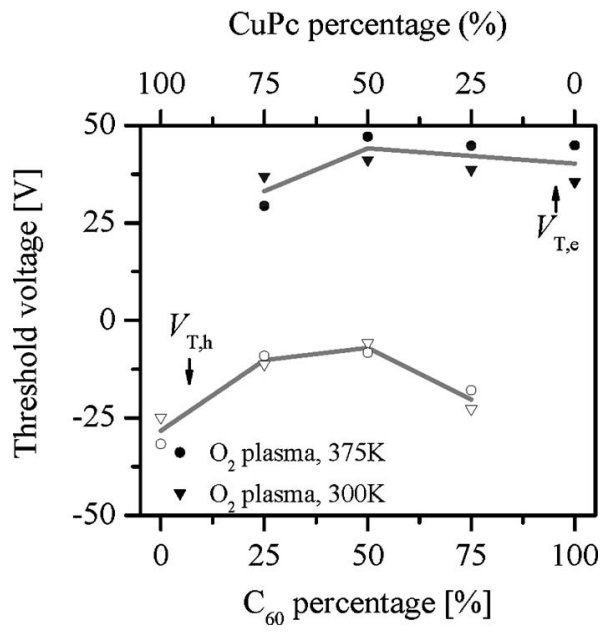

FIG. 7. Dependence of the threshold voltage on the mixing ratio for different temperatures during organic film evaporation (300 and $375 \mathrm{~K}$ ). The threshold voltages are determined from the measurements in the saturation regime. Open symbols are related to the threshold voltages for the holes and filled symbols to the threshold voltages for the electrons. The gray lines are connecting the average values for both treatments and conduce to clarity. 
tion in $\mathrm{C}_{60}$. The change of the threshold voltage for the electron channel is expected to be much weaker than for the hole channel.

The threshold voltage can be related to the work function difference of metal and semiconductor, the doping concentration, the interfacial charge density and the bulk potential. ${ }^{35}$ Considering only interface charges $N_{I f}$, as all other parameters are not expected to change upon mixing of molecules, the threshold voltage shift can be described by

$$
\Delta V_{T}=\frac{e \cdot N_{I f}}{C_{O x}} .
$$

The observed threshold voltage shift of about $21 \mathrm{~V}$ at a mixing ratio of $\mathrm{C}_{60}: \mathrm{CuPc}$ of $1: 1$ results then in an interface charge of 0.024 charges per square nanometer (calculated for a $200 \mathrm{~nm}$ oxide). Corresponding to a charge transfer of 0.012 charges per molecule. This calculation is based on a uniform material distribution at the organic-insulator interface and a $\mathrm{CuPc}$ spacing comparable to the $\alpha$-phase, with approximately two $\mathrm{CuPc}$ molecules per square nanometer. The value mentioned is much less than the reported charge transfer of 0.25 charges per molecule determined from Raman spectroscopy, ${ }^{36}$ however, due to the granular film morphology the calculated value can only be regarded as a crude estimate. Further information concerning the detailed molecular arrangement at the interface to the $\mathrm{SiO}_{2}$ gate dielectric requires a specific quantitative analysis.

\section{Analytical model}

Theoretical studies of ambipolar transport in FET were performed for hydrogenated amorphous silicon $(a-\mathrm{Si}: \mathrm{H}),{ }^{37,38}$ where ambipolar transport was observed for the first time. ${ }^{39}$ Those models were useful for describing measured characteristics but not for the extraction of microscopic transport parameters. More recently, two-dimensional simulations in organic system were performed by Paasch et al. ${ }^{40}$ Their simulations show that ambipolar transport is possible for parameters allowing the injection of both charge carrier types. An analytical model including microscopic transport parameters was developed by Schmechel et al. ${ }^{12}$ It is an extension of the standard Shockley model for inorganic FET and allows for the determination of electron and hole mobilities and the respective threshold voltages. As such, it assumes band transport as in inorganic single-crystalline semiconductors and neglects hopping transport as found in organic systems. The latter problem recently has been treated by Anthopoulos et al. who developed a consistent description for ambipolar OFET based on variable range hopping, including such important features like density dependent charge carrier mobility. ${ }^{41}$ However, for the purpose of this paper the analytical model by Schmechel turned out to be sufficient in order to work out the important features of those different devices.

In this model the drain current of the electrons $\left(I_{D, e}\right)$ and of the holes $\left(I_{D, h}\right)$ is given by ${ }^{12}$
TABLE I. Extracted parameter values (mobility and threshold voltage) for different mixing ratios determined from saturation and the ambipolar regime. Part of the fit curves is also shown in Fig. 4.

\begin{tabular}{lcccc}
\hline \hline & $\begin{array}{c}\mu_{e} \\
\left(\mathrm{~cm}^{2} / \mathrm{V} \mathrm{s}\right) \\
\text { Determined from saturation regime }\end{array}$ & $\begin{array}{c}\mu_{h} \\
\left(\mathrm{~cm}^{2} / \mathrm{V} \mathrm{s}\right)\end{array}$ & $\begin{array}{c}V_{T, e} \\
(\mathrm{~V})\end{array}$ & $\begin{array}{c}V_{T, h} \\
(\mathrm{~V})\end{array}$ \\
\hline${\text { Pure } \mathrm{C}_{60}}$ & $3.2 \times 10^{-1}$ & - & +60.4 & - \\
$\mathrm{C}_{60}: \mathrm{CuPc} 3: 1$ & $2.2 \times 10^{-2}$ & $2.0 \times 10^{-6}$ & +44.8 & -22.7 \\
$\mathrm{C}_{60}: \mathrm{CuPc} 1: 1$ & $1.3 \times 10^{-3}$ & $7.7 \times 10^{-6}$ & +47.4 & -11.9 \\
$\mathrm{C}_{60}: \mathrm{CuPc} 1: 3$ & $3.5 \times 10^{-4}$ & $1.0 \times 10^{-4}$ & +30.7 & -17.0 \\
Pure CuPc & - & $8.5 \times 10^{-3}$ & - & -31.5 \\
\multicolumn{7}{c}{ Determined from ambipolar regime } \\
\hline $\mathrm{C}_{60}: \mathrm{CuPc} 3: 1$ & $2.7 \times 10^{-2}$ & $1.6 \times 10^{-6}$ & +49.5 & -24.0 \\
$\mathrm{C}_{60}: \mathrm{CuPc} 1: 1$ & $1.6 \times 10^{-3}$ & $8.6 \times 10^{-6}$ & +64.5 & -1.7 \\
$\mathrm{C}_{60}: \mathrm{CuPc} 1: 3$ & $3.1 \times 10^{-4}$ & $1.0 \times 10^{-4}$ & +35.1 & -17.7 \\
\hline \hline
\end{tabular}

$$
\begin{aligned}
I_{D, e}= & \frac{W}{2 L} C_{O x} \times\left\{\mu_{e}\left(\llbracket V_{G}-V_{T, e} \rrbracket^{2}-\llbracket\left(V_{G}-V_{T, e}\right)-V_{D} \rrbracket^{2}\right)\right. \\
& \left.+\mu_{h}\left(\llbracket V_{D}-\left(V_{G}-V_{T, h}\right) \rrbracket^{2}\right)\right\},
\end{aligned}
$$

$$
\begin{aligned}
I_{D, h}= & -\frac{W}{2 L} C_{O x} \times\left\{\mu_{h}\left(\llbracket V_{T, h}-V_{G} \rrbracket^{2}-\llbracket V_{D}-\left(V_{G}-V_{T, h}\right) \rrbracket^{2}\right)\right. \\
& \left.+\mu_{e}\left(\llbracket\left(V_{G}-V_{T, e}\right)-V_{D} \rrbracket^{2}\right)\right\} .
\end{aligned}
$$

Therein double brackets are defined as $\llbracket x \rrbracket=\frac{1}{2} x+\frac{1}{2}|x|$ and allow for a full analytical expression instead of a stepwise defined function. The pre-factor includes the specific insulator capacitance $\left(C_{O x}\right)$ and the geometry of the FET ( $W$-channel width and $L$-channel length). The expression in curly brackets contains two parts, one for the normal behavior of a unipolar FET (with $\mu_{e}$ as electron or $\mu_{h}$ as hole mobility) and another one for the quadratic ambipolar increase including the mobility and the threshold voltage of the opposite carrier type. $V_{D}$ and $V_{G}$ are the applied drain and gate voltage, respectively, and $V_{T, e}$ and $V_{T, h}$ the threshold voltages for electrons and holes, respectively. This model is based on homogeneous organic layers and describes both unipolar and ambipolar behavior using transport parameters of both carrier types. It implicitly assumes complete recombination of positive and negative charge carriers.

The model has been used to fit output and transfer characteristics of our samples. As an example, we have included in Fig. 4 the corresponding fit curves from which the respective mobilities and threshold voltages have been derived (see Table I). It is remarkable that the mobilities as determined either from the saturation regime $\left(V_{G}>0, V_{D}>0\right.$ for electrons and $V_{G}<0, V_{D}<0$ for holes, respectively) and from the ambipolar regime $\left(V_{G}>0, V_{D}<0\right.$ for electrons and $V_{G}$ $<0, V_{D}>0$ for holes, respectively) show excellent agreement. Only for the threshold voltages there is a somewhat larger difference between the two regimes. The origin of this phenomenon is still being investigated. Nevertheless, this model has proved to also be very useful for the simulation of ambipolar and complementary inverters as described in the following. 

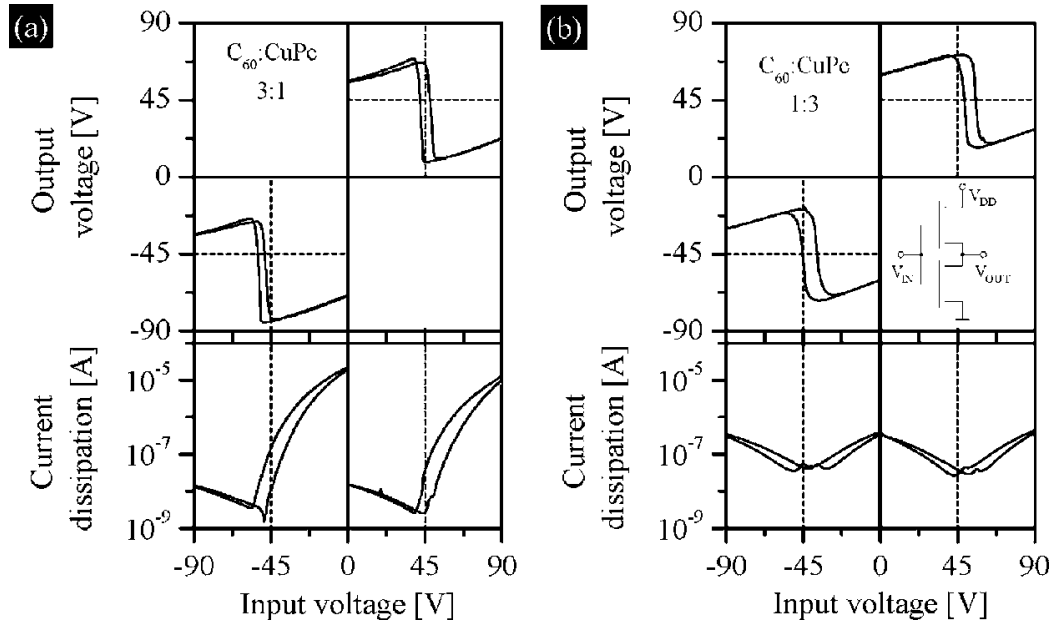

FIG. 8. Characteristics of ambipolar inverters with mixing ratios of 3:1 (a) and 1:3 (b) at a driving voltage $\mathrm{V}_{D D}= \pm 90 \mathrm{~V}$. Transfer characteristics (top) and current dissipation (bottom) are shown. The substrates were $\mathrm{O}_{2}$-plasma treated and the films were evaporated at 375 $\mathrm{K}$ substrate temperature.

\section{E. Measurements of ambipolar and complementary inverters}

As already mentioned, ambipolar transport is involved in various organic semiconductor devices, including photovoltaic cells and light-emitting FET. Additionally there was the suggestion of using ambipolar FET to realize complementary-like organic integrated circuits. ${ }^{7,14}$ Here we investigate ambipolar inverters consisting of mixed-layer ambipolar FET and compare their characteristics to a complementary inverter made of discrete $p$ - and $n$-channel transistors from neat materials.

Figure 8 shows the characteristics of ambipolar inverters using layers with mixing ratios of $3: 1$ and $1: 3$. The upper part presents the transfer characteristics of the inverters (output voltage versus input voltage) and the lower part the corresponding current supplied by $V_{D D}$ which directly gives the dissipated current in the circuit.

A characteristic feature of ambipolar inverters is their operation in the first as well as in the third quadrant of the output-vs.-input diagram, depending on the sign of the supply voltage only. Ideally an inverter should have a sharp transition from the low to the high state at half of the driving voltage and the dissipated current should be negligibly small except for a narrow range around the transition voltage. Both inverters, based on two ambipolar transistors, show this transition at about half of the supply voltage $\left(V_{D D} / 2= \pm 45 \mathrm{~V}\right)$ and reach high gain (about 13 for the 1:3 mixture and about 18 for the 3:1 mixture) which is defined as the steepness of the characteristics at the transitions between the high and the low states. However, they do not reach zero in the low state and the driving voltage in the high state. Also the voltages at the high and the low state are not constant. The noise margin, which is an indicator for the tolerance of cascaded inverter stages, is also high for both inverters (about $14 \mathrm{~V}$ for the $1: 3$ mixture and about $19 \mathrm{~V}$ for the $3: 1$ mixture). Significant differences between the two ambipolar inverters are observed in the dissipated current. Whereas for the inverter with a $1: 3$ mixing ratio the power dissipation is symmetric around $\pm 45 \mathrm{~V}$, the device with a $3: 1$ mixture shows an asymmetry of about three orders of magnitude. Moreover, the dissipated current is as high as $10^{-5} \mathrm{~A}$, which is one-anda-half orders of magnitude larger than in the previous case.
Thus, it is remarkable that a huge asymmetry in electron and hole mobilities of more than three orders of magnitude, as observed for the 3:1 mixture (see Fig. 5), has drastic consequences for the power dissipation of the inverter, although it does not lead to a significant asymmetry in the transition voltage.

For comparison we have also fabricated a complementary inverter by connecting a $p$-channel transistor (pure $\mathrm{CuPc}$ ) and an $n$-channel transistor (pure $\mathrm{C}_{60}$ ) together, its characteristics being shown in Fig. 9. In order to make it operate in the first quadrant it is necessary to connect the $p$-channel transistor to $+V_{D D}$ and the $n$-channel transistor to ground and vice versa the $n$-channel transistor to $-V_{D D}$ and the $p$-channel transistor to ground in order make it operate in the third quadrant. These inverters also show slightly asymmetric transitions with respect to $\pm V_{D D} / 2$ due to the unbalanced electron and hole mobilities in pure $\mathrm{CuPc}$ and $\mathrm{C}_{60}$, but they reach the ground potential in the low state and the supply voltage in the high state. The gain is about 38 and the noise margin between 30 and $50 \mathrm{~V}$. A characteristic differ-

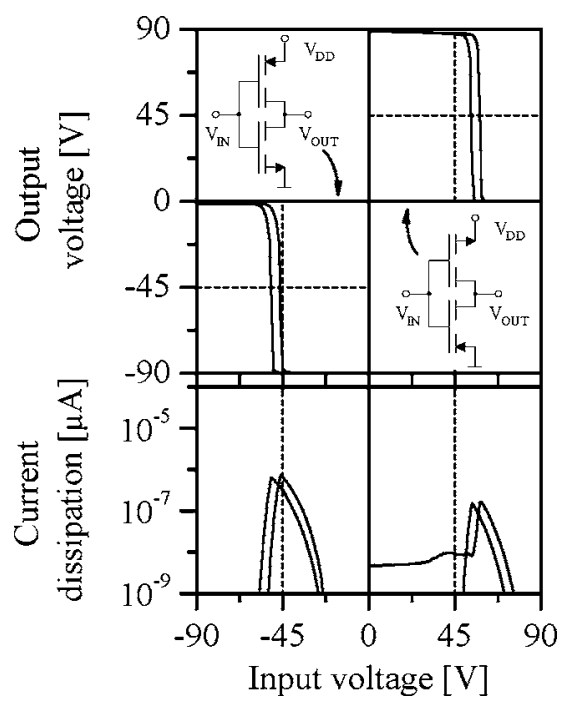

FIG. 9. Transfer characteristics (top) and current dissipation (bottom) of a complementary inverter consisting of discrete $\mathrm{CuPc}$ and $\mathrm{C}_{60}$ transistors. The insets present the circuit and the sign of the driving voltage used to operate the inverter in the first and the third quadrant. The substrate was $\mathrm{O}_{2}$-plasma treated and the film was evaporated at $375 \mathrm{~K}$ substrate temperature. 

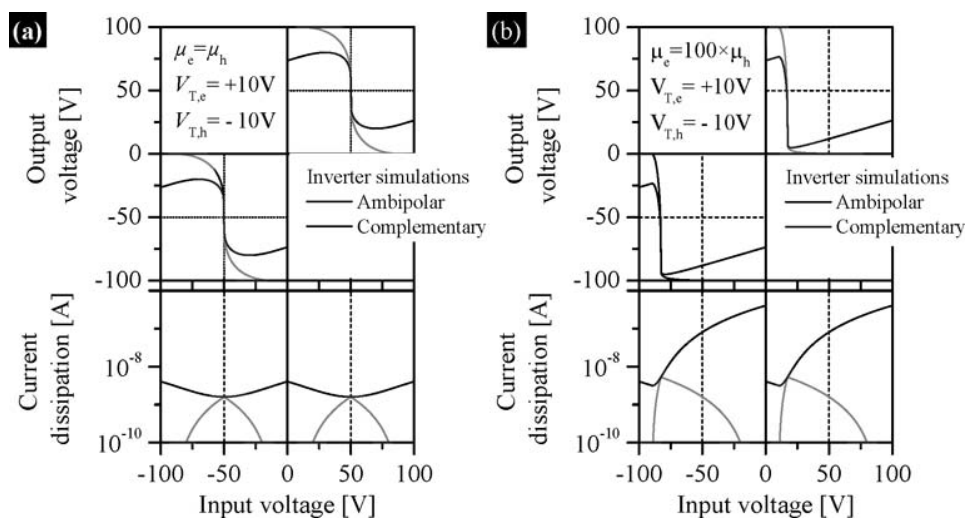

FIG. 10. Simulations of ambipolar and complementary inverter transfer characteristics and current dissipation with symmetric parameters (mobility and threshold voltage) for the $p$ - and the $n$-channel in (a), in comparison to asymmetric mobilities and symmetric threshold voltages in (b).

ence to the ambipolar inverter type is the current dissipation being high only in the vicinity of the transition. This is because at any time one of the two transistors is being switched off in each of the logic states whereas an ambipolar transistor always shows a non-negligible current. We note that the constant current of about $10^{-8} \mathrm{~A}$ which is detected for positive driving voltage between 0 and about $45 \mathrm{~V}$ is not an inverter failure. We believe that this feature is related to the large hysteresis of the $\mathrm{C}_{60}$ FET (see Fig. 2), because it is visible also for other driving voltages and occurs only during decreasing input voltage sweeps.

\section{F. Simulation of ambipolar and complementary inverters}

For a better understanding of inverter characteristics we performed numerical simulations based on the analytical model of Schmechel et al. ${ }^{12}$ [(see Eq. (3)] for both ambipolar and complementary inverters. The output voltage as well as the current dissipation was calculated to demonstrate the differences between ambipolar and complementary inverters and the influence of device parameters like mobility and threshold voltage.

Figure 10 shows simulated transfer characteristics of ambipolar and complementary inverters for balanced and unbalanced charge carrier mobilities. In both cases, as observed before in the measurements, the output voltage of the complementary inverter reaches the driving and the ground voltage in the high and the low state, respectively, whereas the output voltage of the ambipolar inverter does not. Thus the noise margin of the ambipolar inverter is lower than for the complementary inverter. Nevertheless the gain for both types of inverters is comparably high. As expected the current dissipation in the complementary inverter is highest at the transition between the logic levels, whereas the ambipolar inverter has its minimum at this point.

A closer look at the simulations in Fig. 10(a), which are based on symmetric mobilities and threshold voltages, shows that the transition between the logic states is at the half of the driving voltage for both the ambipolar and the complementary inverter. An asymmetry in carrier mobilities [Fig. 10(b)] and/or in threshold voltages (not shown here) leads to a shift of the transition voltage in both cases and a higher current dissipation, particularly for the ambipolar case. This emphasizes the need for balanced electron and hole mobilities in both inverter types.

\section{SUMMARY}

Ambipolar transport in mixed films of $\mathrm{C}_{60}$ and $\mathrm{CuPc}$ was investigated in field-effect transistors and compared to unipolar transport in neat films. Hole and electron transport is observed for all analyzed mixing ratios and deposition temperature with carrier mobilities decreasing exponentially for decreasing volume percentage of the respective conducting material. A mixing ratio of $\mathrm{C}_{60}$ to $\mathrm{CuPc}$ of $1: 3$ is found to yield balanced electron and hole mobilities for all preparation conditions. The variation of the threshold voltage is tentatively explained by a hole accumulation at the $\mathrm{CuPc}$ side of the organic-organic interface.

Furthermore we have demonstrated ambipolar inverters with complementary-like logic behavior from such mixed layers and compared their performance to complementary inverters consisting of discrete $p$ - and $n$-channel transistors of the neat materials. Both our experimental results and the simulations using an analytical model underline the need for balanced carrier mobilities to achieve symmetric inverter characteristics and low losses. While in a complementary inverter this has to be accomplished by a proper choice of $p$ and $n$-conducting materials, the approach using mixed layers, as demonstrated here, allows us to achieve this balance by adjusting the composition of the mixed ambipolar layer.

\section{ACKNOWLEDGMENTS}

This work was supported by the Deutsche Forschungsgemeinschaft (Focus Programme 1121 "Organic Field-Effect Transistors"). The discussed x-ray diffraction measurements were kindly performed by Jens Pflaum (University of Stuttgart).

${ }^{1}$ C. W. Tang and S. A. Vanslyke, Appl. Phys. Lett. 51, 913 (1987).

${ }^{2}$ T. Stübinger and W. Brütting, Proc. SPIE 4465, 102 (2002).

${ }^{3}$ G. Yu, J. Gao, J. C. Hummelen, F. Wudl, and A. J. Heeger, Science 270, 1789 (1995).

${ }^{4}$ J. J. M. Halls, C. A. Walsh, N. C. Greenham, E. A. Marseglia, R. H. Friend, S. C. Moratti, and A. B. Holmes, Nature 376, 498 (1995).

${ }^{5}$ S. Uchida, J. G. Xue, B. P. Rand, and S. R. Forrest, Appl. Phys. Lett. 84, 4218 (2004).

${ }^{6}$ J. Rostalski and D. Meissner, Sol. Energy Mater. Sol. Cells 61, 87 (2000). ${ }^{7}$ E. J. Meijer, D. M. De Leeuw, S. Setayesh, E. Van Veenendaal, B. H. Huisman, P. W. M. Blom, J. C. Hummelen, U. Scherf, and T. M. Klapwijk, Nat. Mater. 2, 678 (2003).

${ }^{8}$ J. Locklin, K. Shinbo, K. Onishi, F. Kaneko, Z. N. Bao, and R. C. Advincula, Chem. Mater. 15, 1404 (2003).

${ }^{9}$ J. Wang, H. B. Wang, X. J. Yan, H. C. Huang, and D. H. Yan, Appl. Phys. Lett. 87, 093507 (2005). 
${ }^{10}$ R. B. Ye, M. Baba, Y. Oishi, K. Mori, and K. Suzuki, Appl. Phys. Lett. 86, 253505 (2005).

${ }^{11}$ J. G. Xue and S. R. Forrest, Phys. Rev. B 69, 245322 (2004).

${ }^{12}$ R. Schmechel, M. Ahles, and H. von Seggern, J. Appl. Phys. 98, 084511 (2005).

${ }^{13}$ L. L. Chua, J. Zaumseil, J. F. Chang, E. C. W. Ou, P. K. H. Ho, H. Sirringhaus, and R. H. Friend, Nature 434, 194 (2005).

${ }^{14}$ T. D. Anthopoulos, S. Setayesh, E. Smith, M. Cölle, E. Cantatore, B. de Boer, P. W. M. Blom, and D. M. de Leeuw, Adv. Mater. 18, 1900 (2006).

${ }^{15}$ G. H. Gelinck, H. E. A. Huitema, E. Van Veenendaal, E. Cantatore, L. Schrijnemakers, J. Van der Putten, T. C. T. Geuns, M. Beenhakkers, J. B. Giesbers, B. H. Huisman et al., Nat. Mater. 3, 106 (2004).

${ }^{16}$ A. Knobloch, A. Manuelli, A. Bernds, and W. Clemens, J. Appl. Phys. 96, 2286 (2004).

${ }^{17}$ A. Dodabalapur, B. Crone, Y. Y. Lin, R. W. Filas, Z. Bao, A. LaDuca, R. Sarpeshkar, H. E. Katz, and W. Li, Nature 403, 521 (2000).

${ }^{18}$ C. Rost, S. Karg, W. Riess, M. A. Loi, M. Murgia, and M. Muccini, Appl. Phys. Lett. 85, 1613 (2004).

${ }^{19}$ M. A. Loi, C. Rost-Bietsch, M. Murgia, S. Karg, W. Riess, and M. Muccini, Adv. Funct. Mater. 16, 41 (2006).

${ }^{20}$ E. J. Meijer, C. Detcheverry, P. J. Baesjou, E. van Veenendaal, D. M. de Leeuw, and T. M. Klapwijk, J. Appl. Phys. 93, 4831 (2003).

${ }^{21}$ N. Marjanovic, T. B. Singh, G. Dennler, S. Gunes, H. Neugebauer, N. S. Sariciftci, R. Schwodiauer, and S. Bauer, Org. Electron. 7, 188 (2006).

${ }^{22}$ M. Knupfer and H. Peisert, Phys. Status Solidi A 201, 1055 (2004).

${ }^{23}$ O. V. Molodtsova, T. Schwieger, and M. Knupfer, Appl. Surf. Sci. 252, 143 (2005).

${ }^{24}$ S. C. Veenstra and H. T. Jonkman, J. Polym. Sci., Part B: Polym. Phys. 41, 2549 (2003).

${ }^{25}$ O. V. Molodtsova and M. Knupfer, J. Appl. Phys. 99, 053704 (2006).

${ }^{26}$ I. Hill, A. Kahn, Z. Soos, and R. Pascal, Chem. Phys. Lett. 327, 181
(2000).

${ }^{27}$ R. W. Lof, M. A. Vanveenendaal, B. Koopmans, H. T. Jonkman, and G. A. Sawatzky, Phys. Rev. Lett. 68, 3924 (1992).

${ }^{28}$ B. P. Rand, J. G. Xue, S. Uchida, and S. R. Forrest, J. Appl. Phys. 98, 124902 (2005).

${ }^{29}$ D. Faiman, S. Goren, E. A. Katz, M. Koltun, N. Melnik, A. Shames, and S. Shtutina, Thin Solid Films 295, 283 (1997).

${ }^{30}$ M. Stöhr, T. Wagner, M. Gabriel, B. Weyers, and R. Möller, Adv. Funct. Mater. 11, 175 (2001).

${ }^{31}$ S. M. Tuladhar, D. Poplavskyy, S. A. Choulis, J. R. Durrant, D. D. C. Bradley, and J. Nelson, Adv. Funct. Mater. 15, 1171 (2005).

${ }^{32}$ V. D. Mihailetchi, L. J. A. Koster, P. W. M. Blom, C. Melzer, B. de Boer, J. K. J. van Duren, and R. A. J. Janssen, Adv. Funct. Mater. 15, 795 (2005).

${ }^{33}$ F. Dinelli, R. Capelli, M. A. Loi, M. Murgia, M. Muccini, A. Facchetti, and T. J. Marks, Adv. Mater. 18, 1416 (2006).

${ }^{34}$ F. Dinelli, M. Murgia, P. Levy, M. Cavallini, F. Biscarini, and D. M. de Leeuw, Phys. Rev. Lett. 92, 116802 (2004).

${ }^{35}$ S. Scheinert and G. Paasch, Phys. Status Solidi A 201, 1263 (2004).

${ }^{36}$ G. Ruani, C. Fontanini, M. Murgia, and C. Taliani, J. Chem. Phys. 116, 1713 (2002).

${ }^{37}$ K. Y. Chung and G. W. Neudeck, J. Appl. Phys. 62, 4617 (1987).

${ }^{38}$ G. W. Neudeck, K. Y. Chung, and H. F. Bare, IEEE Trans. Electron Devices 34, 866 (1987).

${ }^{39}$ G. W. Neudeck and A. K. Malhotra, J. Appl. Phys. 46, 239 (1975).

${ }^{40}$ G. Paasch, T. Lindner, C. Rost-Bietsch, S. Karg, W. Riess, and S. Scheinert, J. Appl. Phys. 98, 084505 (2005).

${ }^{41}$ E. C. P. Smits, T. D. Anthopoulos, S. Setayesh, E. van Veenendaal, R. Coehoorn, P. W. M. Blom, B. de Boer, and D. M. de Leeuw, Phys. Rev. B 73, 205316 (2006). 